\title{
Implication of duration of clinical presentation on tumor progression and short-term recurrence in patients with early breast cancer
}

\author{
TAKAAKI FUJII, REINA YAJIMA, HIROKI MORITA, TOSHINAGA SUTO, \\ HIRONORI TATSUKI, SOICHI TSUTSUMI and HIROYUKI KUWANO \\ Department of General Surgical Science, Graduate School of Medicine, Gunma University, \\ Maebashi, Gunma 371-8511, Japan
}

Received February 16, 2015; Accepted March 20, 2015

DOI: $10.3892 / \mathrm{mco} .2015 .538$

\begin{abstract}
Breast cancer growth is dependent on time and it may be of utmost importance to take into consideration the duration of clinical symptoms in order to predict which patients are at high risk for disease recurrence. The aim of this study was to determine the association between duration of clinical symptoms and disease recurrence in patients with breast cancer. A total of 139 consecutive patients with primary breast cancer who underwent a radical breast operation were retrospectively investigated and the association between recurrence and the duration of symptoms was investigated. The duration of clinical signs was defined as the time from the onset of symptoms to the date of surgery. The breast cancer cases were divided into two groups on the basis of symptom duration ( $\leq 6$ and $>6$ months). The mean duration of symptoms was $191.0 \pm 242.6$ days. Of the 139 cases, 36 (25.9\%) had a duration of symptoms of $>6$ months. In the univariate analysis, a statistically significant association with long symptom duration was observed for disease recurrence. Of the 139 patients, $6(4.3 \%)$ developed recurrent disease. The univariate analysis revealed that long duration of symptoms and nuclear grade were significantly associated with recurrence. In conclusion, our results indicated that breast cancer progression is dependent on time. A long duration of symptoms ( $>6$ months) may be considered as an indicator of tumor progression and a strong prognostic factor in breast cancer patients.
\end{abstract}

\section{Introduction}

An accurate determination of poor prognostic factors for breast cancer patients may help guide the selection of more aggressive adjuvant treatment protocols. The disease stage at

Correspondence to: Dr Takaaki Fujii, Department of General Surgical Science, Graduate School of Medicine, Gunma University, 3-39-22 Showa-machi, Maebashi, Gunma 371-8511, Japan

E-mail: ftakaaki@gunma-u.ac.jp

Key words: breast cancer, duration of symptoms, delay, prognosis diagnosis is widely recognized as one of the most important prognostic factors in patients with breast cancer. Traditionally, the pathological determination of tumor size and lymph node status have guided prognosis and adjuvant therapy recommendations for patients with breast cancer (1-3). Several biomarkers, including endocrine hormone receptor status and human epidermal growth factor receptor 2 (HER2) status, have recently been added to risk evaluation and therapeutic assessment $(4,5)$. However, these assessments are useful for the determination of the disease status at diagnosis or at treatment initiation. Breast cancer is a heterogeneous disease and its natural history exhibits significant variability $(4,6)$. Breast cancer growth is dependent on time; however, certain cancers grow rapidly, whereas others develop slowly. The duration of symptoms is not currently incorporated into the internationally recognized staging systems. However, it may be of utmost importance to take into consideration the duration of clinical symptoms in order to identify patients at high risk for disease recurrence. The majority of patients with breast cancer are detected after the onset of symptoms, rather than through screening $(7,8)$. The effect of the duration of the interval between symptom onset and treatment initiation on progression and survival remains uncertain and cannot be investigated in randomized controlled trials. In this study, we retrospectively investigated the association between the duration of clinical symptoms and disease recurrence in patients with operable breast cancer.

\section{Patients and methods}

Patient assessment. The cases of 139 consecutive patients with primary breast cancer who underwent a radical breast operation at the Department of General Surgical Science, Gunma University Hospital (Gunma, Japan), between January, 2008 and December, 2013 were retrospectively investigated. Patients with previously diagnosed breast cancer or incomplete clinical information and male patients were excluded. None of the patients had received preoperative chemotherapy. The resection margins were clear in all the cases. Informed consent was obtained from all the patients and the study protocol was approved by the Institutional Review Board of the Gunma University Hospital. 

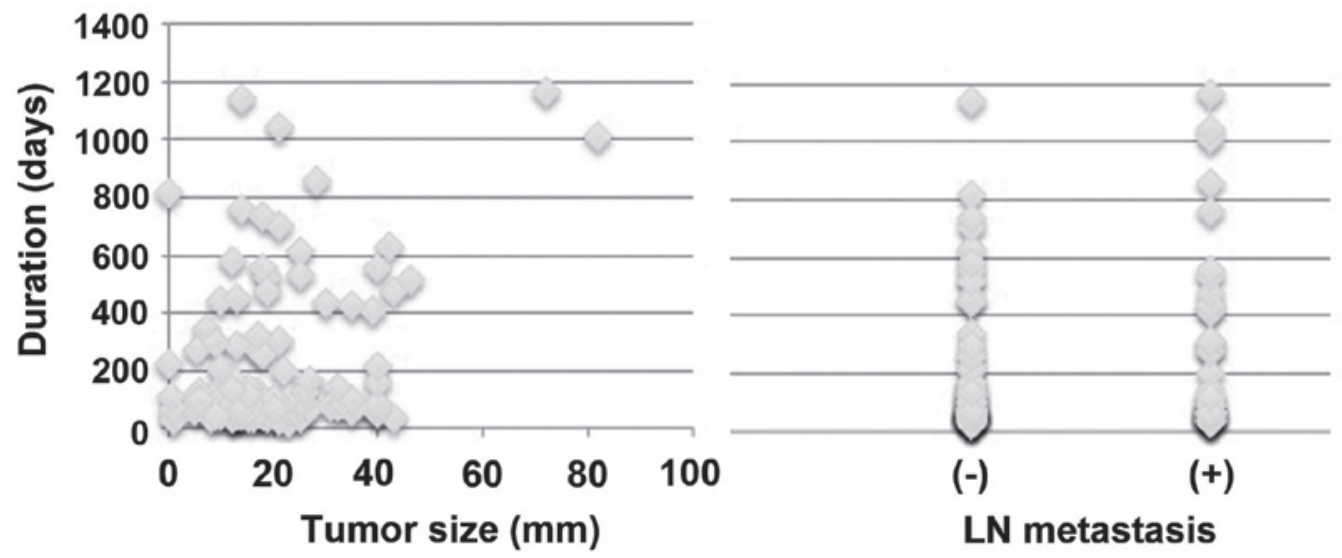

Figure 1. Association of symptom duration with tumor size and lymph node (LN) metastasis. The associations of long symptom duration with tumor size and LN metastasis when using the Pearson's correlation were linear $(\mathrm{R}=0.335, \mathrm{P}<0.05$ and $\mathrm{R}=0.144, \mathrm{P}=0.048$, respectively).

Table I. Association of patient and clinicopathological characteristics with the duration of symptoms.

\begin{tabular}{|c|c|c|c|}
\hline \multirow[b]{2}{*}{ Characteristics } & \multicolumn{2}{|c|}{ Duration } & \multirow[b]{2}{*}{ P-value } \\
\hline & $\begin{array}{l}<6 \text { months } \\
(\mathrm{n}=103)\end{array}$ & $\begin{array}{l}>6 \text { months } \\
\quad(\mathrm{n}=36)\end{array}$ & \\
\hline Age, years ${ }^{\mathrm{a}}$ & $58.9 \pm 12.3$ & $56.0 \pm 12.5$ & 0.235 \\
\hline Histology, no. & & & 0.361 \\
\hline IDC & 90 & 30 & \\
\hline Other & 13 & 6 & \\
\hline Tumor size, $\mathrm{mm}^{\mathrm{a}}$ & $18.1 \pm 9.9$ & $24.0 \pm 17.7$ & 0.992 \\
\hline $\begin{array}{l}\text { Lymph node } \\
\text { metastasis, no. }\end{array}$ & 29 & 16 & 0.112 \\
\hline ER & 86 & 30 & 0.583 \\
\hline $\operatorname{PgR}$ & 80 & 25 & 0.445 \\
\hline HER 2 & 15 & 7 & 0.831 \\
\hline Lymphatic invasion, no. & 37 & 19 & 0.115 \\
\hline Vascular invasion, no. & 14 & 3 & 0.307 \\
\hline Nuclear grade, no. & & & 0.163 \\
\hline $1 / 2$ & 67 & 18 & \\
\hline 3 & 36 & 18 & \\
\hline Recurrence, no. & 2 & 4 & 0.039 \\
\hline
\end{tabular}

The data extracted from the database included age, histological type, primary tumor size, nuclear grade, lymph node metastasis, lymphatic or vascular invasion, estrogen (ER) or progesterone (PgR) status and HER2 score of the primary tumor and patient-reported duration of signs of breast cancer. The ER and PgR status was assessed by the Allred scoring system, with an Allred score of $\geq 3$ defined as ER- and/or PgR-positive $(9,10)$. The duration of clinical symptoms was defined as the time from the onset of symptoms to the date of
Table II. Association of patient and clinicopathological characteristics with disease recurrence.

\begin{tabular}{lccc}
\hline & \multicolumn{2}{c}{ Recurrence } & \\
\cline { 2 - 3 } Characteristics & $\begin{array}{c}\text { Absent } \\
(\mathrm{n}=133)\end{array}$ & $\begin{array}{c}\text { Present } \\
(\mathrm{n}=6)\end{array}$ & P-value \\
\hline Age, years & & & \\
Histology, no. & $58.4 \pm 12.2$ & $54.2 \pm 12.0$ & 0.792 \\
$\quad$ IDC & 114 & 4 & 0.956 \\
Other & 19 & 2 & \\
Tumor size, mm ${ }^{\mathrm{a}}$ & $19.5 \pm 12.6$ & $22.1 \pm 12.7$ & 0.311 \\
Lymph node & 43 & 2 & 0.634 \\
metastasis, no. & & & \\
ER & 113 & 3 & 0.057 \\
PgR & 102 & 3 & 0.157 \\
HER2 & 19 & 2 & 0.224 \\
Lymphatic invasion, no. & 52 & 4 & 0.178 \\
Vascular invasion, no. & 17 & 0 & 0.450 \\
Nuclear grade, no. & & & 0.032 \\
1/2 & 84 & 1 & \\
3 & 49 & 5 & \\
Duration $>6$ months, no. & 4 & 32 & 0.039 \\
\hline
\end{tabular}

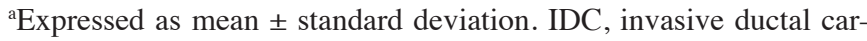
cinoma; ER, estrogen receptor; PgR, progesterone receptor; HER2, human epidermal growth factor receptor 2 .

surgery. The overall median follow-up period was 2.46 years; there was no reported surgical complication-related mortality.

Statistical analysis. The breast cancer cases were divided into two groups on the basis of the symptom duration ( $\leq 6$ and $>6$ months). A univariate statistical analysis was conducted using the Fisher's exact test or the $\chi^{2}$ test, with or without Yates's correction. The Student's t-test was used to compare the two groups. To determine the independence of the risk factors, the 
variables were entered into a multivariate logistic regression model with a likelihood of $\mathrm{P}<0.05$. To investigate possible associations between the duration of symptoms and tumor progression, we computed the Pearson coefficient of correlation and performed a linear regression analysis of the data. $\mathrm{P}<0.05$ was considered to indicate a statistically significant difference.

\section{Results}

Symptom duration is associated with tumor progression in breast cancer. We analyzed the cases of 139 patients with primary breast cancer who underwent elective breast surgery. The mean age of the patients was $58.2 \pm 12.3$ years, with an age distribution of 32-85 years. The mean duration of symptoms was $191.0 \pm 242.6$ days and the median duration of symptoms was 82 days, with a distribution of 20-1,168 days. Of the 139 cases, $36(25.9 \%)$ had a symptom duration of $>6$ months. The patient characteristics and the results of the univariate analysis conducted to determine the association between the duration of symptoms and the clinical variables are summarized in Table I. The univariate analysis revealed a statistically significant association between long duration of symptoms and disease recurrence (Table I). The associations of long symptom duration with tumor size and lymph node metastasis when using the Pearson's correlation were linear $(\mathrm{R}=0.335, \mathrm{P}<0.05$ and $\mathrm{R}=0.144, \mathrm{P}=0.048$, respectively) (Fig. 1). In summary, we were able to establish a connection between long duration of symptoms and tumor progression, including recurrence, lymph node metastasis and tumor size.

Long symptom duration affects the risk of recurrent disease. The breast cancer patients were divided into two groups based on the presence of disease recurrence. Of the 139 patients, $6(4.3 \%)$ exhibited recurrent disease. The patient characteristics and the results of the univariate analysis conducted to determine the association between the clinicopathological variables and disease recurrence are summarized in Table II. The univariate analysis revealed that long symptom duration and nuclear grade were statistically significantly associated with recurrence.

\section{Discussion}

The natural history and clinical relevance of breast cancer vary significantly. In view of tumor progression, in addition to staging or biomarker levels, the natural history of the tumor may have the potential to better define the biological nature of the disease process, regarding risk as well as therapy. In this study, a long duration of symptoms was found to be associated with tumor progression, including tumor size, lymph node metastasis and disease recurrence, in patients with breast cancer. These results suggest that the progression of breast cancer is dependent on time. Thus, a long duration of symptoms may be considered as an indicator of tumor progression and may be a significant prognostic factor in breast cancer. These findings are consistent with the consensus reached by previous studies, namely that longer delays in treatment are associated with larger tumor size and more advanced stage $(5,11,12)$.

The practical significance of these findings is that reducing the delay in presentation and treatment may lead to improved prognosis of patients who present with symptomatic breast cancer. It has been demonstrated that patients with cancer detected upon screening exhibit an improved overall survival and reduced recurrent disease compared with patients with cancers detected following the onset of symptoms $(13,14)$. Effective strategies should be developed for early detection of breast cancer, in order to reduce the duration of symptoms. However, the effect of a symptom duration of $<6$ months is likely to be limited.

A major concern with studies investigating the effect of delay on survival is that the potential confounding effect of lead time bias is not taken into consideration. For patients who eventually succumb to cancer, the interval between treatment and death is shorter if treatment is initiated later in the course of the disease (5). In this study, we investigated cases of symptomatic breast cancer undergoing a radical breast operation. Longer delays between symptom onset and treatment were found to adversely affect survival, which may be attributed to greater tumor progression at treatment initiation. The tumors of patients who experience longer delays may be less biologically aggressive (5); however, tumor grade was associated with longer symptom duration in this study. Further investigation is required to determine whether patients with less aggressive disease experience a longer duration of symptoms.

This study has several potential limitations. The major limitation is that retrospective methods of data collection were used. In addition, the number of cases was relatively limited and the 'true' duration of symptoms and natural cancer history could not be determined. However, the clinical implications of the obtained data are valuable due to the difficulty of large randomized trials with estimating treatment delay. Further investigation is required to overcome the obstacles in designing a prospective study and to determine the significance of symptom duration for prognosis and development of metastatic disease.

In conclusion, the present findings suggest that breast cancer progression is dependent on time and that symptom duration may be a strong prognostic factor in breast cancer patients. A longer duration of symptoms may be considered an indicator of tumor progression and such patients may require more aggressive adjuvant therapies due to the higher risk of distant recurrence.

\section{Acknowledgements}

The authors would like to thank Y. Saitoh, T. Yano, Y. Matsui, A. Ishida and A. Ishikubo for their secretarial assistance. This study was supported in part by Grants-in-Aid from the Japanese Ministry of Education, Culture, Sports, Science and Technology (T.F.).

\section{References}

1. Woodward WA, Strom EA, Tucker SL, et al: Changes in the 2003 American Joint Committee on Cancer staging for breast cancer dramatically affect stage-specific survival. J Clin Oncol 21: 3244-3248, 2003.

2. Taghian A, Jeong JH, Mamounas E, Anderson S, Bryant J, Deutsch M and Wolmark N: Patterns of locoregional failure in patients with operable breast cancer treated by mastectomy and adjuvant chemotherapy with or without tamoxifen and without radiotherapy: Results from five National Surgical Adjuvant Breast and Bowel Project randomized clinical trials. J Clin Oncol 22: 4247-4254, 2004. 
3. Fujii T, Yanagita Y, Fujisawa T, Hirakata T, Iijima M and Kuwano H: Implication of extracapsular invasion of sentinel lymph nodes in breast cancer: Prediction of nonsentinel lymph node metastasis. World J Surg 34: 544-548, 2010.

4. Barginear MF, Bradley T, Shapira I and Budman DR: Implications of applied research for prognosis and therapy of breast cancer. Crit Rev Oncol Hematol 65: 223-234, 2008.

5. Richards MA, Smith P, Ramirez AJ, Fentiman IS and Rubens RD: The influence on survival of delay in the presentation and treatment of symptomatic breast cancer. Br J Cancer 79: 858-864, 1999.

6. Senkus E, Cardoso F and Pagani O: Time for more optimism in metastatic breast cancer? Cancer Treat Rev 40: 220-228, 2014.

7. Burgess CC, Ramirez AJ, Richards MA, Love SB: Who and what influences delayed presentation in breast cancer? Br J Cancer 77 1343-1348, 1998.

8. MacArthur C and Smith A: Delay in breast cancer and the nature of presenting symptoms. Lancet 1: 601-603, 1981.

9. Allred DC, Harvey JM, Berardo M and Clark GM: Prognostic and predictive factors in breast cancer by immunohistochemical analysis. Mod Pathol 11: 155-168, 1998.
10. Shousha S: Oestrogen receptor status of breast carcinoma: Allred/H score conversion table. Histopathology 53: 346-347, 2008.

11. Love RR, Duc NB, Baumann LC, Anh PT, To TV, Qian Z and Havighurst TC: Duration of signs and survival in premenopausal women with breast cancer. Breast Cancer Res Treat 86: 117-124, 2004.

12. Richards MA, Westcombe AM, Love SB, Littlejohns P and Ramirez AJ: Influence of delay on survival in patients with breast cancer: A systematic review. Lancet 353: 1119-1126, 1999.

13. Ahmed M and Douek M: The management of screen-detected breast cancer. Anticancer Res 34: 1141-1146, 2014.

14. Joensuu H, Lehtimäki T, Holli K, Elomaa L, Turpeenniemi-Hujanen T, Kataja V, Anttila A, Lundin M, Isola J and Lundin J: Risk for distant recurrence of breast cancer detected by mammography screening or other methods. JAMA 292: 1064-1073, 2004. 